\title{
Simple Additives Make Polar Fluids More Polar
}

\section{The strong polar nature of bonded molecular pairs called zwitterions boosts the dielectric constants of polar fluids.}

\author{
By Rachel Berkowitz
}

S oft materials with high dielectric constants could increase the performance of energy devices such as capacitors and solid-state batteries. These materials become polarized when subjected to an applied electric field. But many of these materials-for example, polymer electrolyte fluids that conduct only one type of ion-have low dielectric constants. Now, Ralph Colby and his colleagues at Pennsylvania State University show experimentally that they can substantially increase the dielectric constant of polar fluids by adding to them a type of molecule that responds strongly to external electric fields [1].

Mixing a polar solvent and a polymer fluid is the workhorse approach to increasing a polymer's dielectric constant. Doing so, however, reduces the polymer's mechanical strength and lowers the operation temperature of a battery containing this polymer. As such, researchers are searching for alternative additives. One that has attracted attention is a group of molecules known as zwitterions, which have both positively

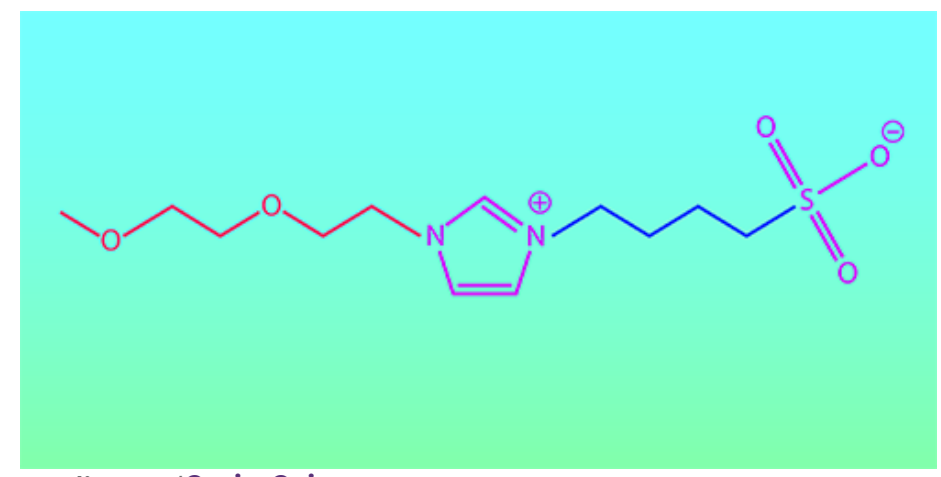

Credit: APS/Carin Cain and negatively charged functional groups.

In spectroscopic studies, the team examined the dielectric constant of six zwitterions in their pure states and mixed in polar fluids. They determined that the molecules' large dipoles increased the polarity of the fluid up to eightfold, realizing dielectric constants as high as 270 at ambient temperature. The team says that using the molecules could provide a new approach for developing energy materials.

Rachel Berkowitz is a Corresponding Editor for Physics based in Vancouver, Canada.

\section{REFERENCES}

1. W. Mei et al., "Zwitterions raise the dielectric constant of soft materials," Phys. Rev. Lett. 127, 228001 (2021). 